\title{
Hydrosystèmes et enjeux de gestion
}

Hydrosystem and management issues

\section{Claire Lang Delus}

\section{(2) OpenEdition}

\section{Journals}

\section{Édition électronique}

URL : http://journals.openedition.org/rge/3487

DOI : $10.4000 /$ rge.3487

ISSN : 2108-6478

\section{Éditeur}

Association des géographes de l'Est

\section{Édition imprimée}

Date de publication : 23 août 2011

ISSN : 0035-3213

\section{Référence électronique}

Claire Lang Delus, "Hydrosystèmes et enjeux de gestion », Revue Géographique de l'Est [En ligne], vol. 51 / 3-4 | 2011, mis en ligne le 28 août 2012, consulté le 22 septembre 2020. URL : http:// journals.openedition.org/rge/3487 ; DOI : https://doi.org/10.4000/rge.3487

Ce document a été généré automatiquement le 22 septembre 2020

Tous droits réservés 


\title{
Hydrosystèmes et enjeux de gestion
}

\author{
Hydrosystem and management issues
}

\section{Claire Lang Delus}

1 La Revue Géographique de l'Est publie un numéro consacré aux hydrosystèmes et aux enjeux liés à leur gestion. Le terme d'«hydrosystème", utilisé à l'origine par les géomorphologues, garde encore une connotation de dynamique fluviale, mais renvoie aujourd'hui à un concept plus vaste et très employé par les géographes. Cette notion a en effet connu une évolution thématique depuis son apparition dans les années 60 , puisqu'elle englobe désormais de nombreuses approches et ouvre le champ de la pluridisciplinarité. L'échelle spatiale des hydrosystèmes se diversifie et l'échelle temporelle prend de plus en plus d'importance, notamment dans l'étude des réponses des hydrosystèmes face aux perturbations naturelle et anthropique. Les différents articles qui composent ce numéro permettent d'illustrer ces tendances, en abordant les hydrosystèmes sous des angles variés et à différentes échelles spatio-temporelles.

2 Les deux premiers articles s'intéressent aux morphologies fluviales et traitent des relations longitudinales et latérales des systèmes fluviaux. Jeannine Corbonnois et al. s'attachent ainsi à décrire les morphologies fluviales et leurs dynamiques, à partir d'un travail de terrain (description de l'état des lits, profils en travers) et d'une analyse de l'évolution des lits sur quelques décennies (interprétation de photographies aériennes et de cartes anciennes). Les auteurs appliquent une analyse statistique multivariée aux descripteurs des formes fluviales, afin d'identifier des indicateurs de la morphogenèse, et proposer un modèle régional de l'état et du fonctionnement des lits fluviaux. Les cours d'eau étudiés, situés dans le Nord-Est de la France, présentent ainsi un contexte régional favorable à la stabilité. Les instabilités ponctuelles s'expliquent par des conditions locales naturelles, ou, le plus souvent anthropiques. Dans un contexte où la dynamique actuelle est essentiellement dictée par les interventions humaines, l'analyse de l'évolution des lits fluviaux proposée par les auteurs constitue une indication de premier ordre sur le temps de réaction des cours d'eau pour s'adapter à de nouvelles conditions.

3 Pour leur part, Jean-Louis Ballais et al. s'intéressent aux unités qui composent la plaine alluviale, en particulier le lit majeur exceptionnel. Les auteurs proposent de faire le 
point sur l'état des connaissances de cette forme fluviale qui n'a été reconnue que récemment. A partir de 10 exemples situés en France et en Tunisie, ils apportent ainsi des précisions intéressantes sur les caractéristiques de cette forme fluviale et les raisons de l'ignorance de son existence, qu'ils assignent à une probable confusion avec les terrasses alluviales. Comme le soulignent les auteurs, toute la difficulté réside dans l'établissement de critères rigoureux qui permettraient de différencier ces deux formes fluviales. Or, dans une optique de cartographie des zones inondables par la méthode hydrogéomorphologique, problématique au sein de laquelle est apparue l'existence du lit majeur exceptionnel, il apparaît essentiel de pouvoir interpréter ces formes.

4 La problématique du risque inondation est abordée, sous un autre angle, dans l'article suivant. Bertrand Sajaloli et al. s'intéressent en effet à la prise en compte du risque inondation dans les politiques urbaines de trois villes ligériennes. Dans cet article, les auteurs classent les paysages urbains situés en zone inondable en trois types qui distinguent des espaces créés, recomposés ou ignorés par la prise en compte du risque inondation dans les politiques urbaines. Ils proposent, pour chaque catégorie, un indice de résilience grâce à une grille d'analyse paysagère fondée sur des marqueurs paysagers de la résilience. Cette grille d'évaluation paysagère permet de produire une cartographie de la résilience urbaine face au risque inondation. Le poids des marqueurs de la résilience dans la notation finale présente l'inconvénient d'être déterminé de manière qualitative, mais, comme le soulignent les auteurs, cette difficulté pourrait être palliée par des analyses statistiques multifactorielles qui permettraient d'évaluer ce poids de manière quantitative. La méthode développée présente l'avantage d'être transposable à l'ensemble des villes ligériennes, fournissant ainsi un outil comparatif des politiques urbaines en matière d'intégration du risque inondation.

5 L'article suivant porte sur la problématique des hydrosystèmes lacustres. Pascal Bartout s'intéresse en effet à l'évolution spatiale des retenues d'eau à travers l'étude des archives du cadastre napoléonien. L'auteur mène un intéressant travail d'analyse géohistorique, visant à comprendre la place et l'évolution de ces zones humides au cours du temps, afin d'en adapter la gestion actuelle. Généralement situés en tête des bassins versants, les étangs remplissent en effet de nombreuses fonctions et constituent des enjeux notamment environnementaux dans la mesure où ils impactent la qualité et la quantité des eaux en aval. Dans le contexte actuel de la Directive Cadre Européenne, ces retenues constituent donc de forts enjeux et il apparaitt nécessaire de mieux les connaitre. Pascal Bartout s'appuie ainsi sur une comparaison entre le cadastre napoléonien et les inventaires actuels pour comprendre l'évolution spatiale des retenues d'eau. Il souligne les limites de l'utilisation de telles archives mais met également en exergue les lacunes des inventaires actuels en rappelant la nécessité, dans une logique de gestion de ces hydrosystèmes, de recenser spatialement et de mieux caractériser les étangs.

6 Les deux derniers articles abordent les hydrosystèmes sous l'angle quantitatif de la ressource en eau et de la problématique des étiages. Claire Lang Delus concentre son analyse sur les processus de genèse des étiages. Si ces phénomènes hydrologiques sont essentiellement dépendants des apports pluviométriques, la relation évidente entre un étiage marqué et une pluviométrie faible est loin d'être simple. En effet, les étiages sont des événements à la genèse particulièrement longue et les précipitations doivent donc être observées plusieurs mois à l'avance, en particulier durant la période de recharge des nappes. De plus, d'autres facteurs sont à prendre en considération, notamment les 
capacités aquifères des bassins versants qui conditionnent les apports souterrains et donc le soutien des débits durant la période d'étiage. Une bonne connaissance des processus inhérents à la genèse de ces événements extrêmes permet une anticipation du risque d'étiage, en accord avec les préconisations du plan national sécheresse qui vise une gestion plus cohérente de la ressource en eau en période de crise.

7 Dans un contexte caractérisé par une pression croissante sur la ressource en eau, les étiages font actuellement l'objet de nombreux travaux, d'autant plus que ces événements pourraient, avec le changement climatique, devenir plus graves et plus fréquents. La question de l'impact d'un changement climatique sur les débits d'étiage est posée dans le dernier article. Didier François et al. s'interrogent en effet sur les impacts d'un changement climatique sur les débits d'étiage de la Meuse au cours du XXIème siècle. Ils mènent une démarche prospective, qui consiste à simuler des débits à partir d'un modèle hydrologique alimenté par différents scénarios climatiques. Les auteurs s'attachent à souligner les nombreuses sources d'incertitude qui accompagnent ce type d'étude, la plus importante étant liée à la vraisemblance des scénarios climatiques. Les résultats sont donc à interpréter avec prudence, car comme l'annonce le titre de la contribution, il s'agit d'une " évolution possible des débits ". Il n'en reste pas moins que certains résultats apparaissent préoccupants et devraient encourager des réflexions sur des stratégies d'adaptation.

Les hydrosystèmes sont des milieux complexes, qui interagissent de manière croissante avec les interventions anthropiques. La connaissance de l'évolution de ces systèmes et de leurs fonctionnements passés et actuels permet d'envisager leurs dynamiques futures pour proposer des stratégies de gestion adaptées. C'est tout l'objet de ce numéro qui, à travers l'analyse des hydrosystèmes passés et actuels, et de leurs interactions avec les sociétés, ouvre des perspectives et des pistes de réflexion pour répondre aux enjeux actuels de gestion.

\section{AUTEUR}

\section{CLAIRE LANG DELUS}

Université Nancy 2, CERPA - Centre d'Études et de Recherches sur les Paysages - 23, boulevard Albert I ${ }^{\mathrm{er}}, 54000 \mathrm{NANCY}$, claire.delus@univ-nancy2.fr 